\title{
Emerging 5G Multicarrier Chaotic Sequence Spread Spectrum Technology for Underwater Acoustic Communication
}

\author{
Wu Jinqiu $\mathbb{D}^{1},{ }^{1}$ Qiao Gang, ${ }^{2}$ and Kang Pengbin ${ }^{1}$ \\ ${ }^{1}$ Beijing Institute of Control and Electronic Technology, Beijing 100000, China \\ ${ }^{2}$ College of Underwater Acoustic Engineering, Harbin Engineering University, Harbin 150001, China \\ Correspondence should be addressed to Wu Jinqiu; jinqiuwu@yahoo.com
}

Received 28 February 2018; Accepted 21 June 2018; Published 18 October 2018

Academic Editor: Christos Volos

Copyright (c) $2018 \mathrm{Wu}$ Jinqiu et al. This is an open access article distributed under the Creative Commons Attribution License, which permits unrestricted use, distribution, and reproduction in any medium, provided the original work is properly cited.

\begin{abstract}
Generalized frequency division multiplexing (GFDM) is a newly introduced technique for the wireless fifth-generation (5G) standard based on multicarrier filter bank theory, which has the advantage of flexibility in setting the number of subcarriers and subblocks. The application of GFDM in underwater acoustic (UWA) communication can take full advantage of the limited spectral resources, which is a prime limitation in UWA communication and will promote the development of UWA network technology. However, the multicarrier communication technique utilized in radio 5G communication offers difficulty in channel estimation, and the influence of a channel cannot be ignored especially in the UWA communication field. Therefore, GFDM cannot be implemented directly in UWA communication; to solve this problem, a system combining chaotic sequence spread spectrum technology with GFDM is proposed, which is a novel technique with high spectrum efficiency. Simulation and experimental results verified the effectiveness of the proposed system.
\end{abstract}

\section{Introduction}

Next-generation communication technology is aimed at achieving a higher data transmission rate, and bandwidthlimited UWA communication is no exception. The full utilization of the extremely limited spectrum resources has always been a focus of future research. One of the solutions for this problem, which recently attracted researchers' attention, is the emerging multicarrier scheme GFDM. Unlike OFDM [1], the impulse response and the frequency response of the prototype filter in GFDM can be designed according to particular requirements and there is no need for subcarriers to maintain orthogonality [2-4]. The implementation of GFDM is based on the filter bank theory, which causes trouble in the channel estimation, and to the best of our knowledge, no related research has solved this problem yet; thus, the application of GFDM has been limited to radio communication only. However, the influence of the time-varying frequency-selective UWA channels cannot be ignored $[5,6]$, and the GFDM technology cannot be applied directly to the UWA communication. Therefore, we propose the GFDM-
CSSS (GFDM-chaotic sequence spread spectrum) system which combined GFDM and spread spectrum technologies to overcome the channel estimation problem. The rest of this paper is organized as follows. In Section 2, the emerging multicarrier technology GFDM is introduced in detail. The system model of GFDM-CSSS UWA communication is proposed in Section 3. Then, the simulation and experimental results are given in Section 4. Finally, the work is concluded in Section 5.

\section{Generalized Frequency Division Multiplexing Technology}

GFDM is a two-dimensional (time-domain and frequencydomain) block structure technology based on the filter banks $[7,8]$, which divides the transmitting data into different subblocks and subcarriers, and each subcarrier is filtered through a prototype filter, which has the advantage of low out-of-band leakage and low PAPR. The advantage of freely selecting the number of subcarriers and subblocks allows the technique to utilize fragmented spectral resources and 
to flexibly allocate spectrum resources [3]. Compared with OFDM, GFDM is a flexible multicarrier technology, which will introduce more degrees of freedom [1]. The structure of modulated GFDM data is shown in Figure 1, with each GFDM symbol adding one CP in front of the symbol.

The modulator of GFDM is shown in Figure 2. Firstly, the $N$ spread spectrum data are converted from serialized to paralleled data and then grouped into $K$ groups, and each group contains $M$ subblocks and satisfies the equation $M \times K=N$. Each group transmits on one subcarrier, and finally the modulated data will be combined and transmitted together. The dimension of the modulated data vector is $N \times 1$, which is composed of $K$ subcarriers and $M$ subsymbols.

Modulated data $\vec{d}$ can be expressed as $\vec{d}=\left(\vec{d}_{0}^{T}, \ldots\right.$, $\left.\vec{d}_{M-1}^{T}\right)^{T}, \quad$ in $\quad$ which $\quad \vec{d}_{0}=\left(\vec{d}_{0,0}^{T}, \ldots, \vec{d}_{K-1,0}^{T}\right)^{T}, \quad \vec{d}_{m}=$ $\left(\vec{d}_{0, m}^{T}, \ldots, \vec{d}_{K-1, m}^{T}\right)^{T}$, and $d_{k, m}$ respect the data that transmitted on the $k$-th subcarrier in the $m$-th subsymbol. The corresponding impulse response is $g_{k, m}$, which is shown in

$$
g_{k, m}[n]=g[(n-m K) \bmod N] \cdot \exp \left[-j 2 \pi \frac{k}{K} n\right] .
$$

As can be seen from (1), each $g_{k, m}[n]$ is obtained by the prototype filter through different time and frequency conversions.

The transmitting data $\vec{x}=(x[n])^{T}$ can be expressed as

$$
x[n]=\sum_{k=0}^{K-1} \sum_{m=0}^{M-1} g_{k, m}[n] \cdot d_{k, m}, \quad n=0, \ldots, N-1 .
$$

Let $\vec{g}_{k, m}=\left(g_{k, m}[n]\right)^{T}$; then, (2) can be written as

$$
\vec{x}=A \vec{d}
$$

The dimension of modulation matrix $A$ is $K M \times K M$, which can be written as

$$
A=\left(\vec{g}_{0,0} \ldots \vec{g}_{K-1,0} \vec{g}_{0,1} \ldots \vec{g}_{K-1, M-1}\right) .
$$

$\vec{g}_{k, m}$ is generated by $\vec{g}_{0,0}$ through time- and frequencydomain shifting; for example, $\vec{g}_{1,0}=[A]_{n, 2}$ and $\vec{g}_{0,1}=$ $[A]_{n, K+1}$ are the cyclic shift of $\vec{g}_{0,0}=[A]_{n, 1}$. By adding the $\mathrm{CP}$ to the modulated data $\tilde{\vec{x}}$, the data can be transmitted through the underwater acoustic channel. There are many filters that can be selected in the GFDM communication system. Table 1 gives the frequency response of several typical filters.

In which, $\operatorname{lin}_{\alpha}(x)$ is

$$
\operatorname{lin}_{\alpha}(x)=\min \left(1, \max \left(0,\left(\frac{1+\alpha}{2 \alpha}+\frac{|x|}{\alpha}\right)\right)\right) .
$$

Equation (5) is used to describe the roll-off area in the frequency domain, and $p_{4}(x)=x^{4}\left(35-84 x+70 x^{2}-20 x^{3}\right)$.
Compared with OFDM technology, GFDM utilizes fewer cyclic prefixes and, in consequence, has a higher spectral efficiency. And GFDM technology has more selectable filter banks. GFDM retains all these advantages at the expense of additional implementation complexity. The block structure of GFDM can be designed according to the requirements, especially for systems with limited bandwidth. The flexibility in selecting subblocks and subcarriers allows the GFDM to take full advantage of the scattered spectrum, which greatly improves the spectral efficiency and can be applied to future multiuser underwater acoustic communications. As a multicarrier technology compared with OFDM, one of the biggest advantages of GFDM is the low peak-toaverage ratio (PAPR); [9] gives a detailed PAPR performance of GFDM. However, the structure causes difficulty in channel estimation, which makes it unusable in UWA communication whose channel condition is more complicated than that of radio communications [10-12]. Therefore, we propose a communication system which combines GFDM with spread spectrum technology. This system can take full advantage of the anti-multipath ability of spread spectrum technology and performs well in both simulation and experiment.

\section{System Model of GFDM-CSSS UWA Communication}

Due to the good autocorrelation of the spreading sequence, effective receiving signals can be easily extracted. The spread spectrum communication system includes the process of spread spectrum modulation and despreading, which will effectively separate the strongest useful signal from the multipath signals, so that signal fading due to multipath interference in UWA communications can be effectively eliminated. Therefore, the spread spectrum communication system can perform well without channel estimation.

Spread spectrum technology can be realized in the time domain or frequency domain; however, the frequencydomain spread affects the subcarriers and influences the performance of the system; thus, the time-domain spread spectrum is adopted here. Furthermore, the chaotic sequence is selected among several spread spectrum sequences due to its flexible length selection and a variety of optional sequences. The block diagram of the GFDM-CSSS system is shown in Figure 3. The source data is encoded, and then spectrum is spread by chaotic sequences, followed by GFDM modulation. After adding $\mathrm{CP}$, the data is transmitted into the UWA channel. At the receiving end, the $\mathrm{CP}$ is removed after synchronization; then, the data is demodulated and despread. Spread spectrum and GFDM modulation are two main components at the transmitter; correspondingly, despread spectrum and demodulation are the main components at the receiver. The detailed structures of GFDM-CSSS spread and despread spectrum are shown in Figures 4 and 5, respectively. Despreading is being done by the locally generated chaotic sequence, and the data is obtained after integration and decision. The despreading and decision procedure is discussed in the following. 


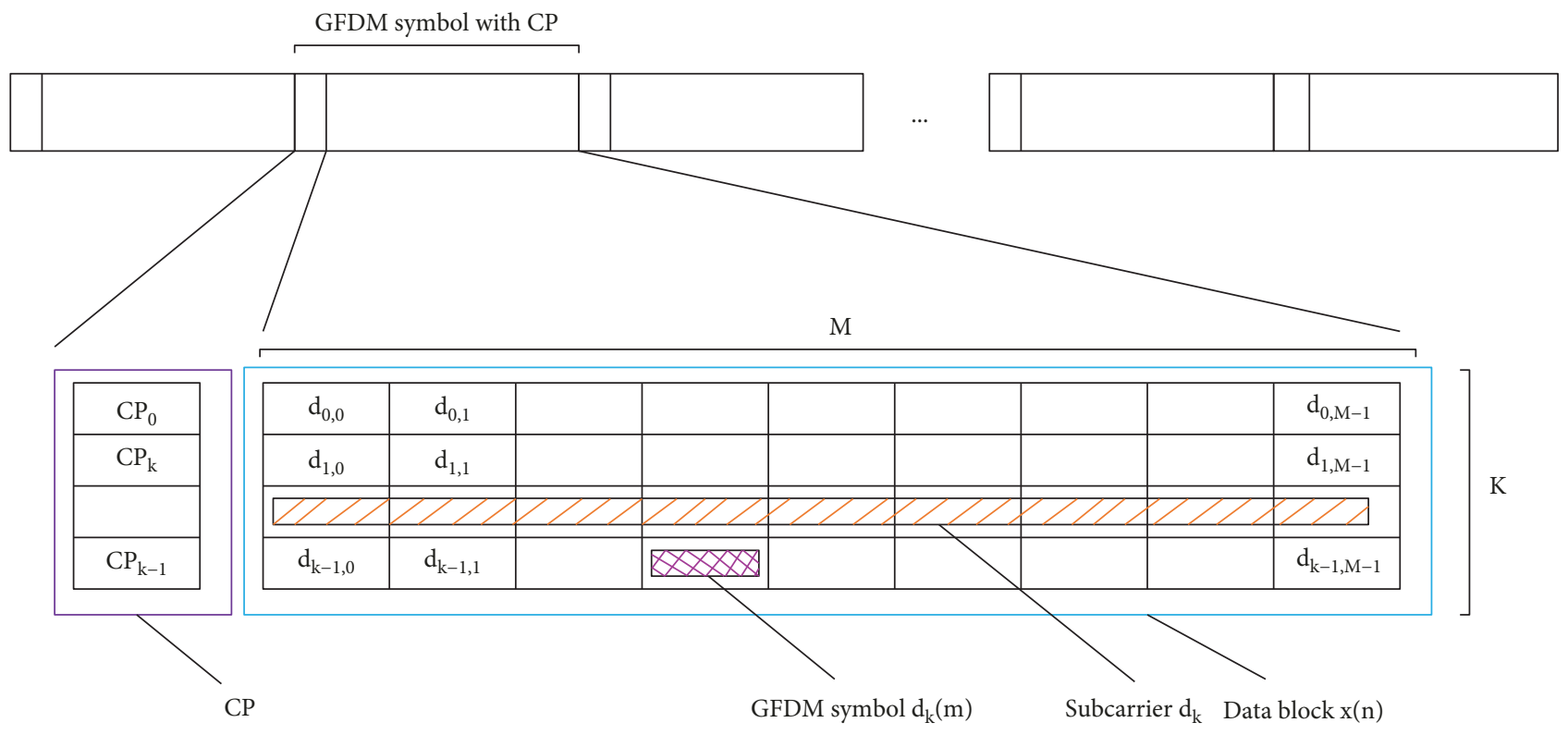

FIgURE 1: The structure of GFDM data block.

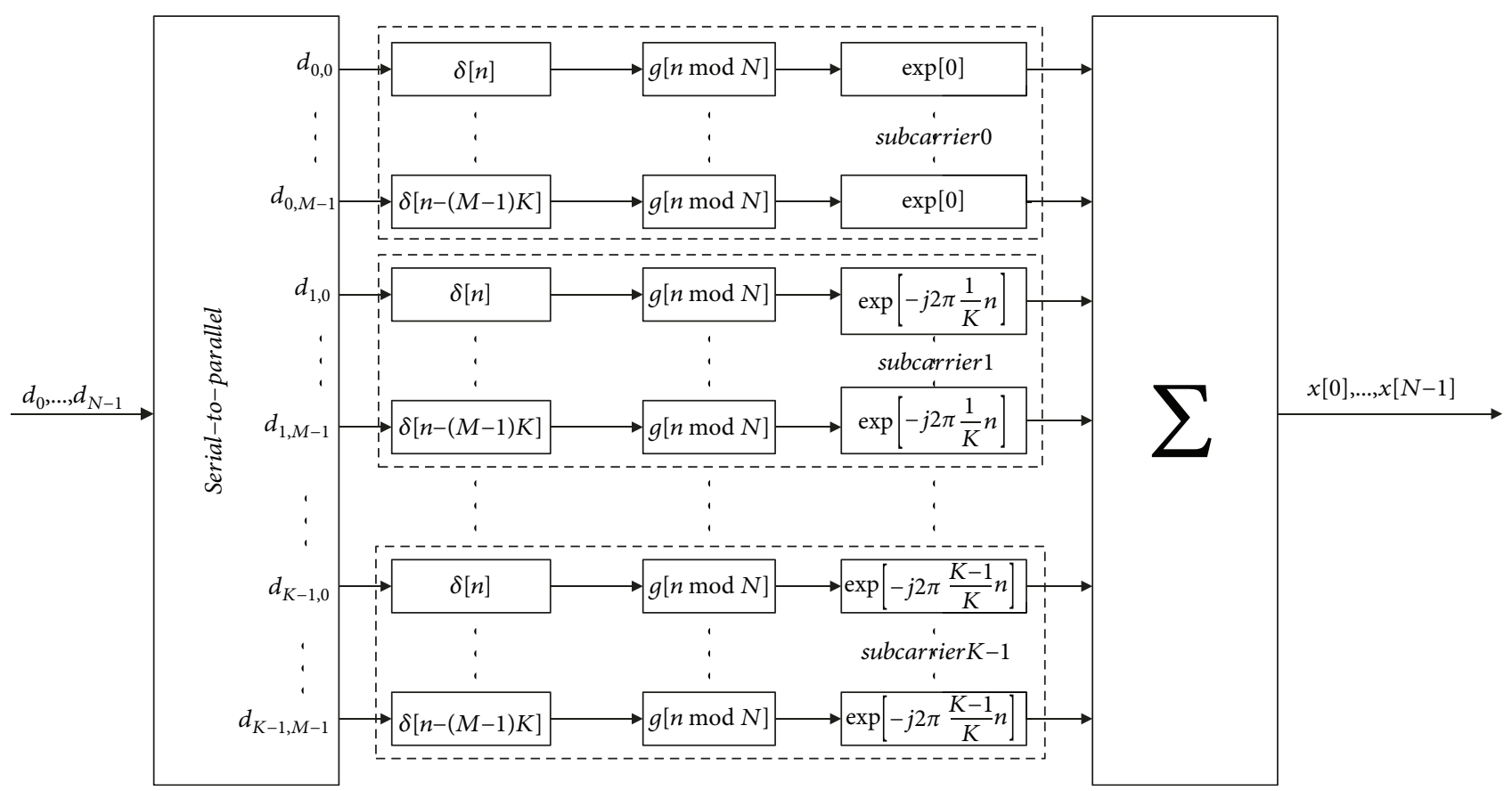

Figure 2: Structure of GFDM modulation.

TABLE 1: Frequency response of different filters.

\begin{tabular}{lc}
\hline Name & Frequency response \\
\hline RC & $G_{\mathrm{RC}}[f]=\frac{1}{2}\left[1-\cos \left(\pi \operatorname{lin}{ }_{\alpha}\left(\frac{f}{M}\right)\right)\right]$ \\
RRC & $G_{\mathrm{RRC}}[f]=\sqrt{G_{\mathrm{RC}}[f]}$ \\
1st Xia & $G_{\mathrm{Xia} 1}[f]=\frac{1}{2}\left[1-\exp \left(-j \operatorname{lin}{ }_{\alpha}\left(\frac{f}{M}\right) \operatorname{sign}(f)\right)\right]$ \\
4th Xia & $G_{\mathrm{Xia} 4}[f]=\frac{1}{2}\left[1-\exp \left(-j \pi p_{4}\left(\operatorname{lin}_{\alpha}\left(\frac{f}{M}\right)\right) \operatorname{sign}(f)\right)\right]$ \\
\hline
\end{tabular}

The GFDM demodulation is represented by $g^{-1}[\cdot]$ which is the inverse process of GFDM modulation. And the demodulation data can be expressed as

$$
\widehat{d}(t)=g^{-1}[z(t)] .
$$

$c_{r}(t)$ is the despread spectrum that resulted from the locally generated spread spectrum sequence which is the 


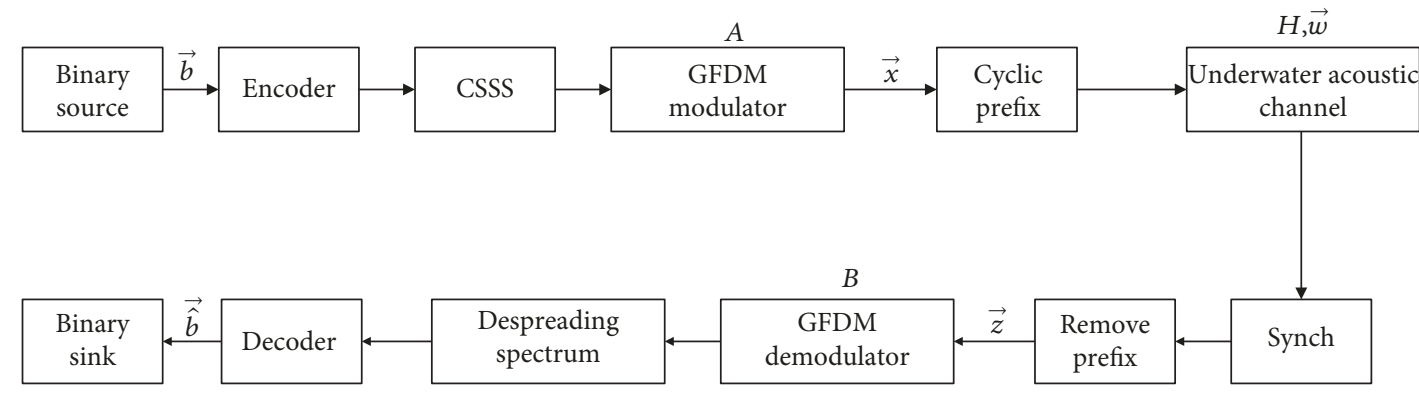

Figure 3: Block diagram of the transceiver.

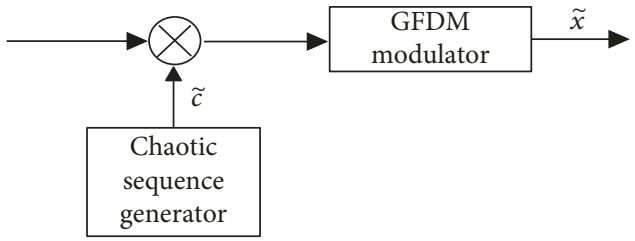

FIGURE 4: Structure of GFDM-CSSS spread spectrum.

same with the transmitting terminal, assuming no effects of the channel; therefore,

$$
\begin{aligned}
m(t) & =\widehat{d}(t) c_{r}(t)=g^{-1}[z(t)] c_{r}(t)=g^{-1}[g[d(t) c(t)]] c_{r}(t) \\
& =d(t) c(t) c_{r}(t) .
\end{aligned}
$$

Integrate $m(t)$ in its duration time:

$$
q(t)=\int_{0}^{T_{b}} m(t) d t
$$

where the pulse duration of the spreading spectrum sequence $c(t)$ is $T_{b}$, which means

$$
\int_{0}^{T_{b}} c(t) c_{r}(t) d t=T_{b}
$$

Therefore, $q(t)$ can be expressed as

$$
q(t)= \begin{cases}T_{b}, & \text { when } d(t)=1, \\ -T_{b}, & \text { when } d(t)=-1 .\end{cases}
$$

Then, the judgment of the signal can be made by judging the positivity and negativity of $q(t)$.

\section{The Simulation and Experiments of the GFDM-CSSS UWA Communication System}

4.1. The Simulation Results. The BER performances for GFDM and GFDM-CSSS systems in the UWA field are compared with different filter banks and rolling-off factors under the condition that the modulation matrix structures are the same (with the number of subblocks $M=2$ and the number of subcarriers $K=29$ ). Take the RC filter as an example, whose frequency response is given in Table 1 .

Figures 6(a) and 6(b) show the BER performances of the GFDM and GFDM-CSSS system in AWGN and multipath channels, which proves that the GFDM technology used in radio communication does not suit the UWA environment. As for the tiny BER difference between GFDM-CSSS systems with different rolling-off factors, it can also be concluded that the rolling-off factor has little effect on the BER performance. The BER comparison of the GFDM-CSSS system with different modulation matrix structures is given in Figure 7. The premise of the comparison is that the total transmit bits are the same. Therefore, two kinds of modulation matrix structure were taken as an example, which are $M=2, K=29$ and $M=29, K=2$, respectively. When BER reaches $10^{-3}$, the $K=2$ structure is obviously better than the $K=29$ structure by about $10 \mathrm{~dB}$ in the AWGN channel. We can conclude that the less the number of subcarriers, the better the BER performance will be. When the number of subcarriers is small, it means that the intercarrier interference is small. Therefore, the BER performance becomes better.

4.2. The Experimental Results. The experiment was carried out in a water pool, which is 25 meters long, 15 meters wide, and 10 meters high and is surrounded by anechoic wedges shown in Figure 8. The working frequency of the transmitting transducer is $3-8 \mathrm{kHz}$, and the laying depth is 3 meters. The horizontal distance between the transmitter and the hydrophone is 11.5 meters. Both of the transducers are shown in Figures 9(a) and 9(b). The maximum multipath delay of the channel is about $5.5 \mathrm{~ms}$ as shown in Figure 10 . Two kinds of filters-RC and RRC-were taken as examples, whose rolling-off factor $\alpha$ equals to 0.1 . Table 2 shows the experimental results of the GFDM system and the proposed system with RC and RRC filters. Both of them are with the modulator structure $M=29, K=2$. The proposed system with the BER equals to 0 which is significantly superior to the GFDM system that is used in the radio communication field. However, the BER performance of the GFDM-CSSS system will reduce with the increase in the number of subcarriers. Therefore, it is necessary to design a reasonable channel estimation method for the GFDM-CSSS system when the subcarrier number is high. 


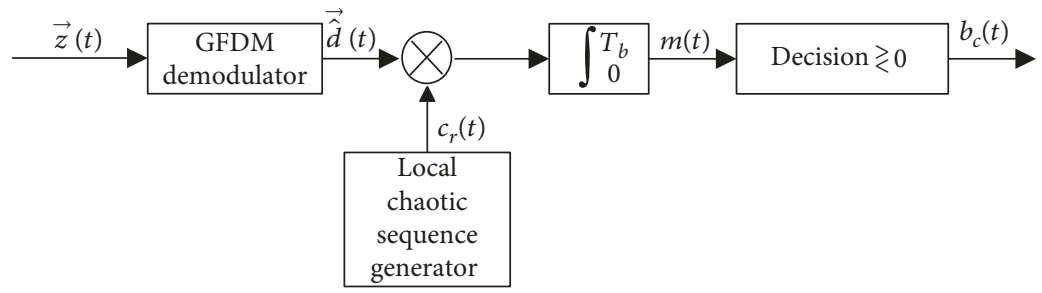

FIGURE 5: Structure of GFDM-CSSS despread spectrum.

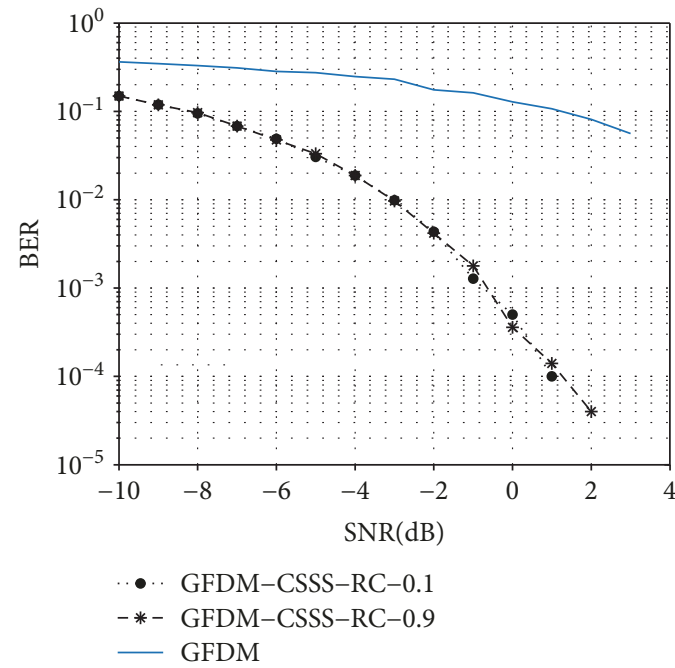

(a)

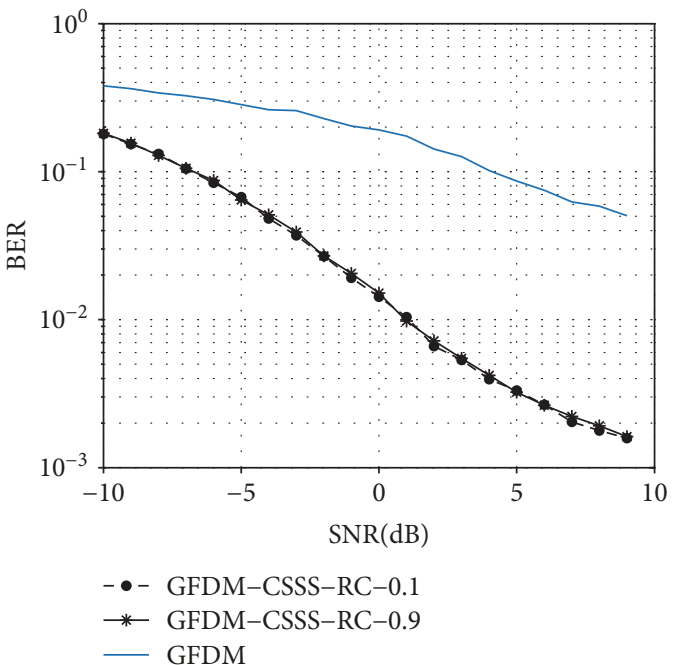

(b)

FIGURE 6: BER performance comparison of GFDM and GFDM-CSSS with different filter banks in the AWGN and multipath channel.

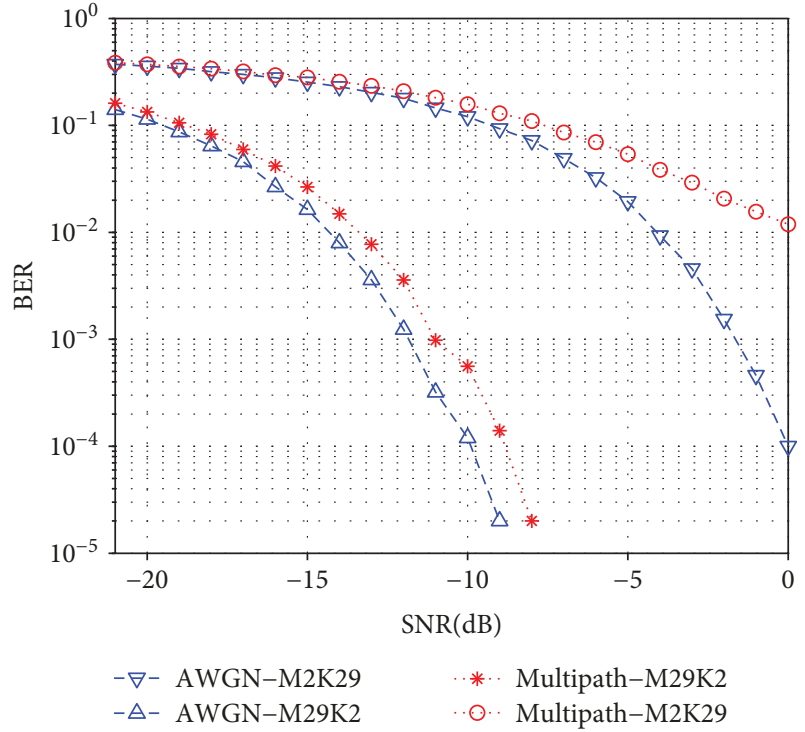

FIGURE 7: BER performance comparison of GFDM-CSSS with different modulation matrix structure in the AWGN and multipath channel.

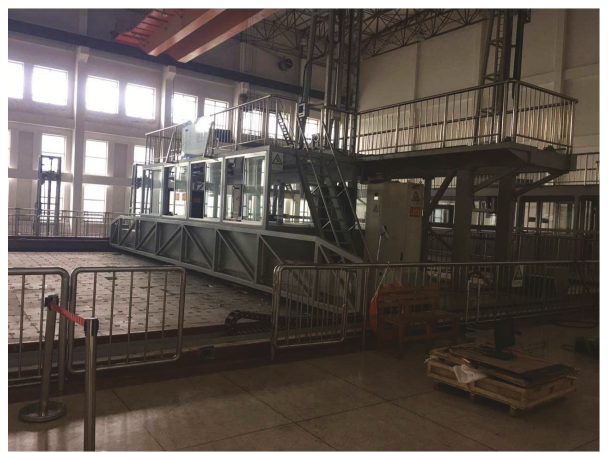

Figure 8: Anechoic tank.

\section{Conclusion}

The GFDM-CSSS communication system proposed in this paper is the extension of the emerging terrestrial 5G multicarrier technology GFDM for underwater acoustic communication for the first time which enhances the popularization of multicarrier underwater acoustic communication and promotes the development of the underwater acoustic network. The flexibility in setting the number of subcarriers and subblocks in GFDM helps in taking full advantage of the sparse spectral resources in UWA communication. Furthermore, 


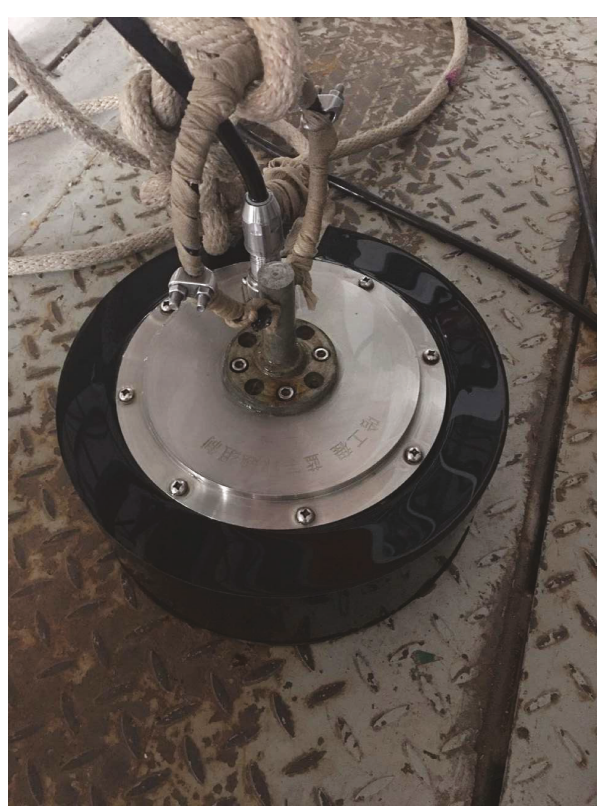

(a)

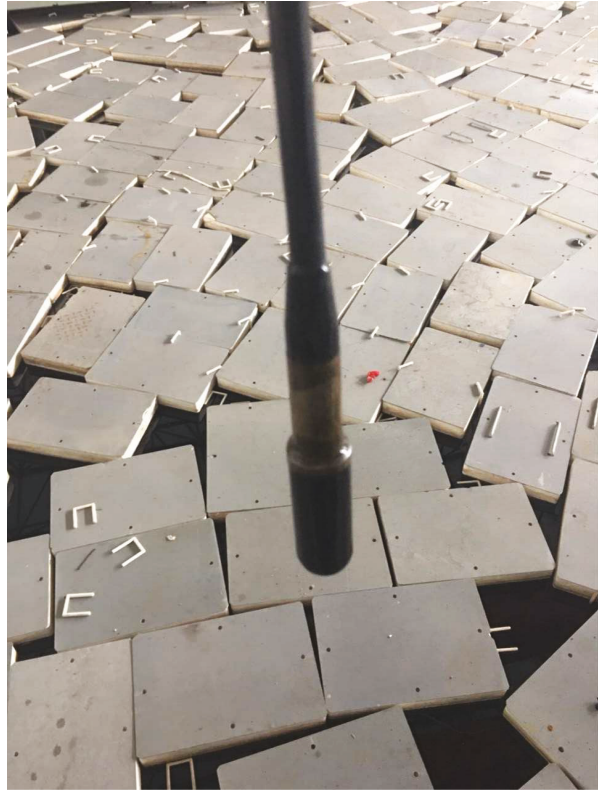

(b)

FIgURe 9: (a) Transmitting transducer. (b) Hydrophone.

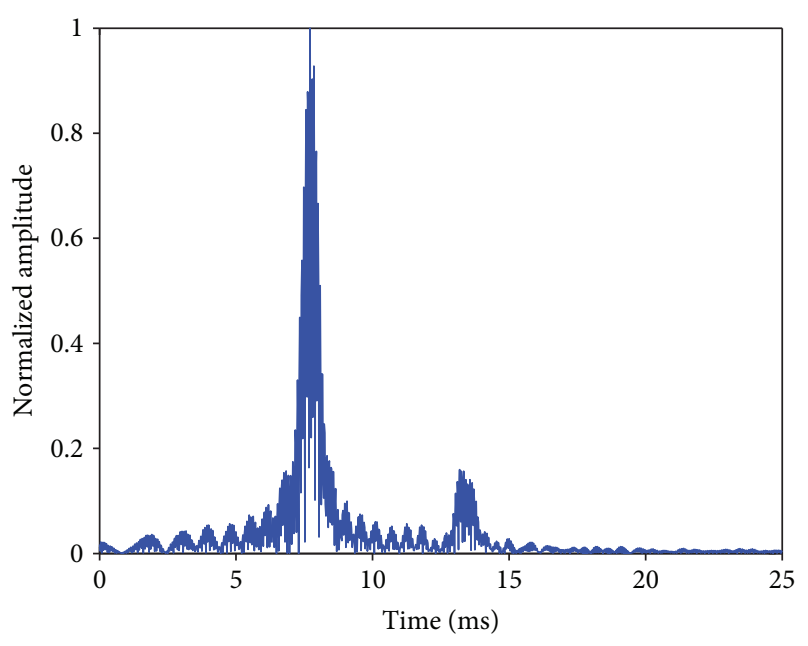

FIgURE 10: Experimental measured channel impulse response.

TABLE 2: BER of the GFDM and GFDM-CSSS system with different filters.

\begin{tabular}{lcc}
\hline \multirow{2}{*}{ Filter } & \multicolumn{2}{c}{ Structure } \\
& $M=2, K=29$ & $M=29, K=2$ \\
BER & BER \\
\hline GFDM-RC filter & 0.3253 & 0.0326 \\
GFDM-RRC filter & 0.3083 & 0.0488 \\
GFDM-CSSS-RC filter & 0.0681 & 0 \\
GFDM-CSSS-RRC filter & 0.0259 & 0 \\
\hline
\end{tabular}

the low PAPR increases the energy efficiency as compared with other multicarrier techniques. Simulation and experimental results verify the effectiveness of the system. However, the BER performance degrades with the increase in the number of subcarriers; therefore, the channel estimation and equalization algorithm applicable to the system with a large number of subcarriers will be studied in the future.

\section{Data Availability}

The data used to support the findings of this study are available from the corresponding author upon request.

\section{Conflicts of Interest}

The authors declare that they have no conflicts of interest.

\section{Acknowledgments}

The authors thank the project of the National Natural Science Foundation of China (nos. 61431004, 61601136, and 61601138) and the Fundamental Research Funds in Heilongjiang Provincial University (no. 135209239). The authors also thank the Technical Bureau of Qiqihar (GYGG201622).

\section{References}

[1] B. Lim and Y. C. Ko, "SIR analysis of OFDM and GFDM waveforms with timing offset, CFO and phase noise," IEEE Transactions on Wireless Communications, vol. 16, no. 10, pp. 6979-6990, 2017.

[2] G. Fettweis and S. Alamouti, "5G: personal mobile internet beyond what cellular did to telephony," IEEE Communications Magazine, vol. 52, no. 2, pp. 140-145, 2014.

[3] G. Wunder, P. Jung, M. Kasparick et al., "5GNOW: nonorthogonal, asynchronous waveforms for future mobile applications," IEEE Communications Magazine, vol. 52, no. 2, pp. 97-105, 2014. 
[4] N. Michailow, I. Gaspar, S. Krone, M. Lentmaier, and G. Fettweis, "Generalized frequency division multiplexing: analysis of an alternative multi-carrier technique for next generation cellular systems," in 2012 International Symposium on Wireless Communication Systems (ISWCS), pp. 171-175, Paris, France, August 2012.

[5] M. Barforooshan and R. Mahboobi Esfanjani, "Stabilization over frequency-selective channels subject to transmission delay and signal-to-noise ratio limitations," Complexity, vol. 21, Supplement 1565 pages, 2016.

[6] G. Fettweis, M. Krondorf, and S. Bittner, "GFDM - generalized frequency division multiplexing," in Proc. IEEE 69th VTC Spring, pp. 1-4, Barcelona, Spain, April 2009.

[7] M. Matthe, N. Michailow, I. Gaspar, and G. Fettweis, "Influence of pulse shaping on bit error rate performance and out of band radiation of generalized frequency division multiplexing," in 2014 IEEE International Conference on Communications Workshops (ICC), pp. 43-48, Sydney, NSW, Australia, June 2014.

[8] M. H. Abbaszadeh, B. H. Khalaj, and A. Haghbin, "Optimum low complexity filter bank for generalized orthogonal frequency division multiplexing," EURASIP Journal on Wireless Communications and Networking, vol. 2018, no. 1, 2018.

[9] J. Wu, X. Ma, X. Qi, Z. Babar, and W. Zheng, "Influence of pulse shaping filters on PAPR performance of underwater 5G communication system technique: GFDM," Wireless Communications and Mobile Computing, vol. 2017, Article ID 4361589, 7 pages, 2017.

[10] S. Han, Y. Sung, and Y. H. Lee, "Filter design for generalized frequency-division multiplexing," IEEE Transactions on Signal Processing, vol. 65, no. 7, pp. 1644-1659, 2017.

[11] M. H. Abbaszadeh, B. H. Khalaj, and A. Haghbin, "Error performance analysis for generalized orthogonal frequency division multiplexing," Computers \& Electrical Engineering, vol. 61, pp. 139-150, 2017.

[12] D. Gaspar, L. Mendes, and T. Pimenta, "GFDM BER under synchronization errors," IEEE Communications Letters, vol. 21, no. 8, pp. 1743-1746, 2017. 


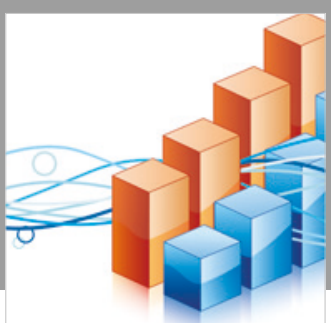

Advances in

Operations Research

\section{-n-m}
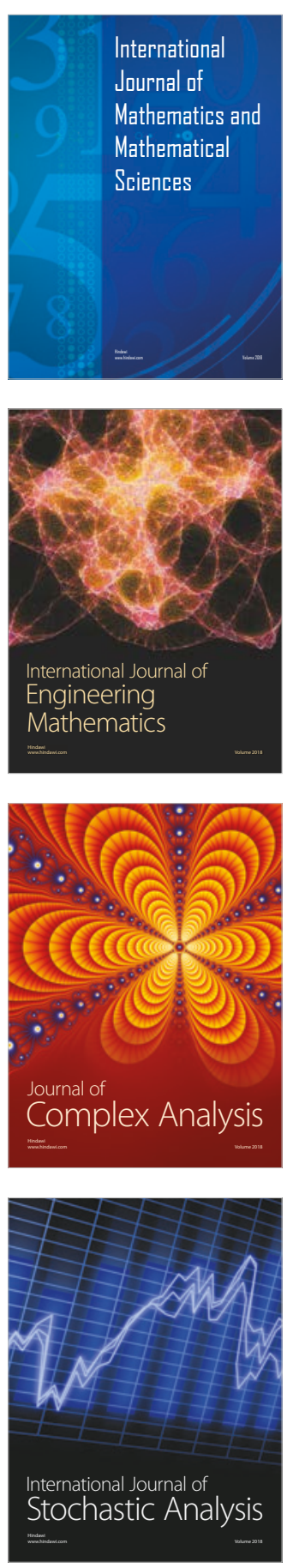
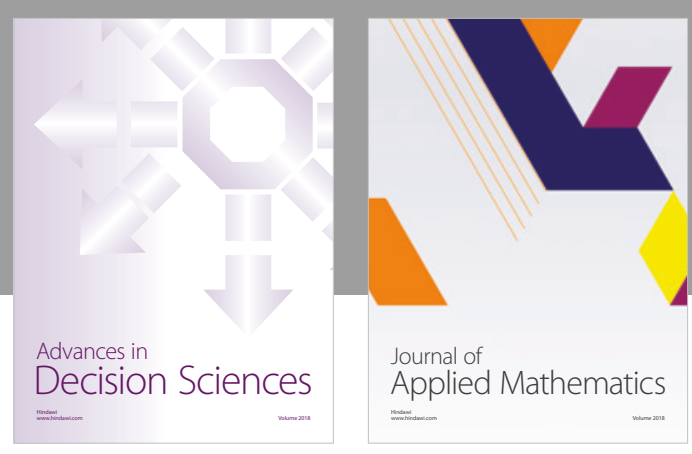

Journal of

Applied Mathematics
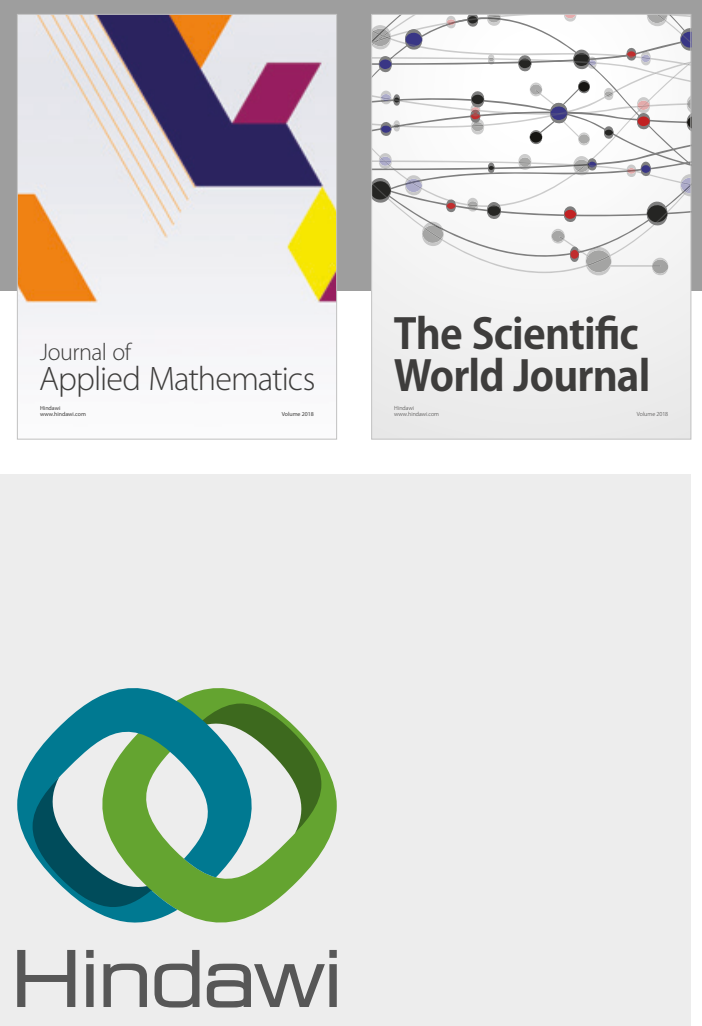

Submit your manuscripts at

www.hindawi.com

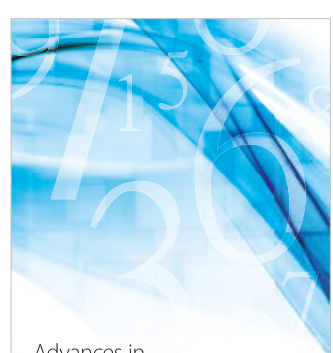

Advances in
Numerical Analysis
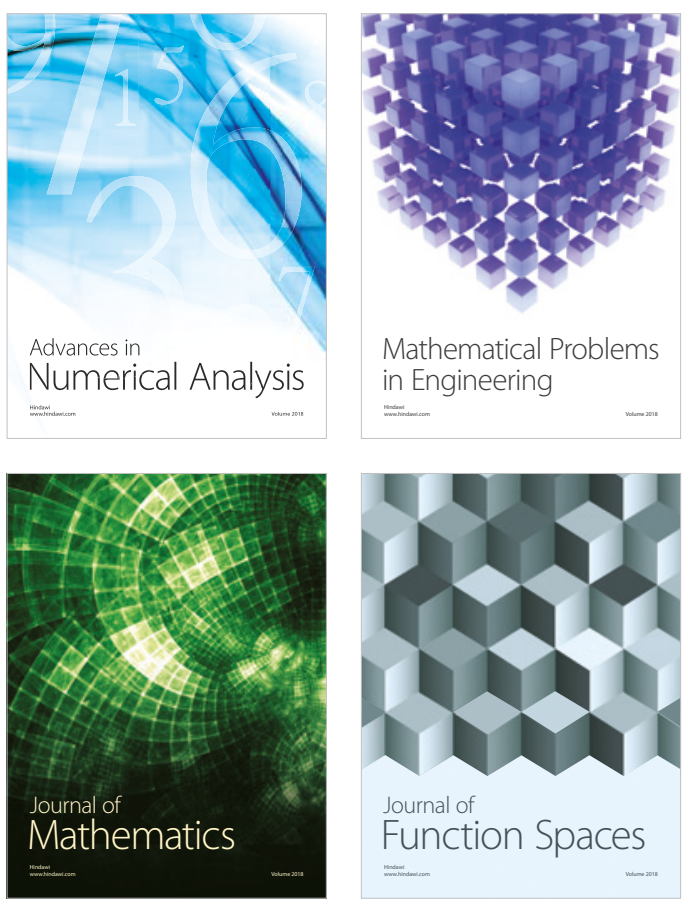

Mathematical Problems in Engineering

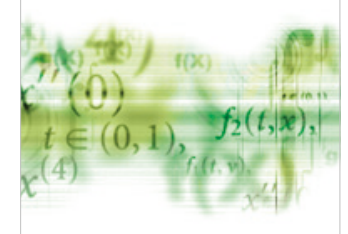

International Journal of

Differential Equations

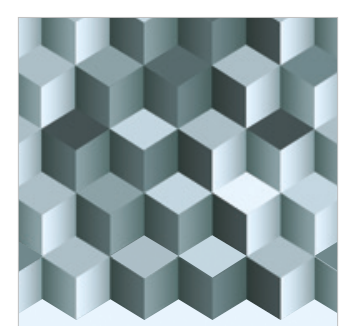

Journal of

Function Spaces
The Scientific

World Journal

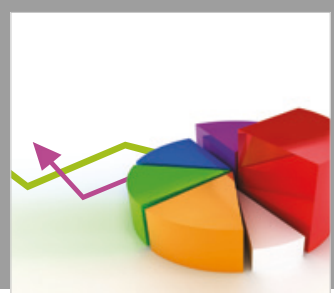

Journal of

Probability and Statistics
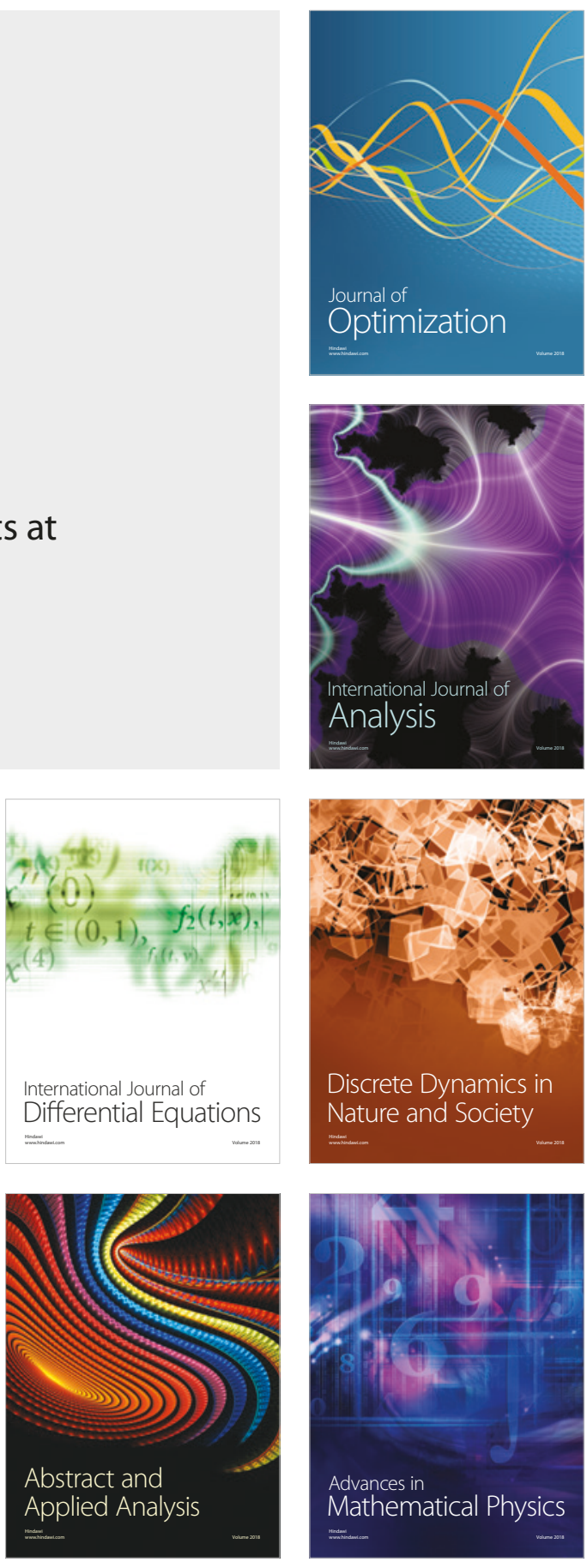